doi:10.13108/2020-12-2-35

\title{
GROWTH OF SUBHARMONIC FUNCTIONS ALONG LINE AND DISTRIBUTION OF THEIR RIESZ MEASURES
}

\author{
A.E. SALIMOVA, B.N. KHABIBULLIN
}

\begin{abstract}
Let $u \not \equiv-\infty$ and $M \not \equiv-\infty$ be two subharmonic functions on a complex plane $\mathbb{C}$ with Riesz measures $\nu_{u}$ and $\mu_{M}$, respectively, such that $u(z) \leqslant O(|z|)$ and $M(z) \leqslant O(|z|)$ as $z \rightarrow \infty$, and $q$ is some positive continuous function on a real axis $\mathbb{R}$, and mes is a linear Lebesgue measure on $\mathbb{R}$. We assume that the following condition for the growth of function $u$ along the imaginary axis $i \mathbb{R}$ of the form
\end{abstract}

$$
u(i y) \leqslant \frac{1}{2 \pi} \int_{0}^{2 \pi} M\left(i y+q(y) e^{i \theta}\right) \mathrm{d} \theta+q(y) \quad \text { for ally } \in \mathbb{R} \backslash E,
$$

where $E \subset \mathbb{R}$ is some small set, for instance, $\operatorname{mes}(E \cap[-r, r]) \leqslant q(r)$ as $r \geqslant 0$. Under such restrictions for the function $u$ it is natural to expect that the Riesz measure $\nu_{u}$ is in some sense majorized by the Riesz measure $\mu_{M}$ of the function $M$ or by integral characteristics of the function $M$. We provide a rigorous quantitative form of such majorizing. The need in such estimates arises naturally in the theory of entire functions in its applications to the completeness issues of exponential systems, analytic continuation, etc. Our results are formulated in terms of special logarithmic characteristics of measures $\nu_{u}$ and $\mu_{M}$ arisen earlier in classical works by P. Malliavin, L.A. Rubel and other for sequences of points and also in terms of special logarithmic characteristics of the behavior of the function $M$ along the imaginary axis and of the function $q$ along the real axis. The obtained results are new also for distribution of the zeroes of entire functions of exponential type under restrictions for the growth of such function along a line. The latter is demonstrated by a new uniqueness theorem for entire functions of exponential type employing so-called logarithmic block-densities of the distribution of the points on the complex plane.

Keywords: subharmonic function of a finite type, Riesz measure, entire function of exponential type, distribution of zeroes, uniqueness theorem

Mathematics Subject Classification: 31A05, 30D20, 30D15

\section{INTRODUCTION}

1.1. Main problem and origination. Let $u \not \equiv-\infty$ and $M \not \equiv-\infty$ be subharmonic functions of finite type (of order 1 ) on the complex plane $\mathbb{C}$, which means the finitness of the type

$$
\text { type }[u]:=\limsup _{z \rightarrow \infty} \frac{u(z)}{|z|}
$$

and type type $[M]<+\infty$ with Riesz measures $\nu_{u}:=\frac{1}{2 \pi} \Delta u$ and $\mu_{M}:=\frac{1}{2 \pi} \Delta M$, respectively, where $\Delta$ is Laplace operator acting in the sense of generalized functions [1], 2]. We assume that the growth of the function $u$ along some straight line $L \subset \mathbb{C}$ is majorized by the function

A.E. Salimova, B.N. Khabibullin, Growth of subharmonic functions along Line And DistriBUTION OF THEIR RIESZ MEASURES.

(C) Salimova A.E., Khabibullin B.N. 2020.

The Research is FinAnCially Supported By the Grant of Russian Science Foundation (Project NO. 18-11-00002).

Submitted November 26, 2019. 
$M$ or, more generally, by some means of the function $M$ over circumferences with centers in $L$, with certain additive terms to $M$ and not everywhere in $L$ but outside some exceptional set $E \subset L$. In this case it is natural to expect that the Riesz measure $\nu_{u}$ should be majorized somehow by the Riesz measure $\mu_{M}$ related with the radii of the above circumference, with the characteristics of additive term and the smallness of the exceptional set $E$. Our main aim is to provide quantitative characteristics of such majorizing the measure $\nu_{u}$ by the measure $\mu_{M}$ in terms of special logarithmic characteristics/densities of the measures $\nu_{u}$ and $\mu_{M}$. Theorem 1 formulated below in Section 1.3 as well as its variations, Proposition 1, Corollary 1, Theorem 2 in Section 3 are new results even for a special choice $u=\ln |f|$ and $M=\ln |g|$ in the case of entire functions of exponential type $f \neq 0$ and $g \neq 0$ with type $[\ln |f|]<+\infty$ and type $[\ln |g|]<+\infty$, as the Riesz measures $\nu_{u}$ and $\mu_{M}$ the sequences of zeroes or roots Zero $f$ and Zero $g$ of respectively functions $f$ and $g$ serve; these zeroes are taken in some order counting their multiplicities. For entire functions of exponential type we establish a final uniqueness theorem 3 .

Exactly in formulation for entire functions of exponential type $f$ and $g$, a version of our main problem was considered in joint work by P. Malliavin and L.A. Rubel [3], in which as the straight line $L$, the imaginary axis $i \mathbb{R} \subset \mathbb{C}$ served, where $\mathbb{R} \subset \mathbb{C}$ was the real axis. We follow the same choice. In [3], for an arbitrary entire function of exponential type $g$ with zeroes in the right half-plane $\mathbb{C}_{\mathrm{rh}}:=\{z \in \mathbb{C}: \operatorname{Re} z>0\}$ located exceptionally on real semi-axis $\mathbb{R}^{+}:=\{x \in \mathbb{R}: x \geqslant 0\}$, there was provided a complete description of all positive sequences of the points $\mathbf{Z}=\left\{\mathbf{z}_{k}\right\}_{k=1,2, \ldots} \subset \mathbb{R}^{+}$, for each of them there exists an entire function of exponential type $f \neq 0$ vanishing on $Z$ and obeying the constraint $\ln |f(i y)| \leqslant \ln |g(i y)|$ for all $y \in \mathbb{R}$. To this problem, one of the main section in the joint monograph by L.A. Rubel and J.E. Colliander was devoted [4, Sect. 22]. In the series of works by the second author in 1988-1991, all these results were extended for arbitrary sequences complex sequences $\mathbf{Z} \subset \mathbb{C}$ with an upper bound $\ln |f(i y)| \leqslant M(i y)$ for all $y \in \mathbb{R}$ via special subharmonic majorizing function $M$ instead of $\ln |g|$, namely, for an arbitrary small $\varepsilon>0$ for $M(z)=\varepsilon|z|, z \in \mathbb{C}$ originally in the paper by I.F. Krasichkov-Ternovskii [5, Thms. 8.3, 8.5, Cor. 5.6] only for sequences $Z$ in the vicinity of $i \mathbb{R}$; for arbitrary $\mathbf{Z} \subset \mathbb{C}$ in [6, Main thm.] with an addition in [7, Main thm., Thm. 1], and also in much more general form with the majorant of form $M(z)=\ln |g(z)|+\varepsilon|z|$, where $g \neq 0$ is an entire function of exponential type in [8, Thm. 1] and in [9, Main thm.], or even more generally and strictly, with majorants of $M=\ln |g|$ with $\varepsilon=0$ but for the sequences $\mathbf{Z} \subset \mathbb{C}$ separated by a pair of vertical angles from the imaginary axis in [10, Main thm.]. The situation with a subharmonic majorizing function $M$ of a finite type and order 1 harmonic in a pair of vertical angles containing $i \mathbb{R} \backslash\{0\}$ was to some extent studied in the thesis of the second co-author [11, Ch. II], but in scientific journals the latter results with arbitrary subharmonic majorant $M$ of a finite type were not published. The most part of the above results were presented in the monograph [12, 3.2] with detailed historical comments.

1.2. Notations and definitions. In this subsection we provide preliminary information needed for formulation our main theorem 1. We denote by sbh the set of all subharmonic functions on $\mathbb{C}$ and $\operatorname{sbh}_{*}:=\{u \in$ sbh : $u \neq \equiv-\infty\}$.

According the context, by the same symbol 0 we denote the number zero, the zero function, zero measure, etc.; $\varnothing$ is the empty set. Everywhere the positivity is treated as $\geqslant 0$, and negativity is $\leqslant 0$.

The symbol Meas ${ }^{+}$stands for the class of all positive Borel measures on $\mathbb{C}$, mes is the linear Lebesgue measure on $\mathbb{R}, C(X)$ is the class of all continuous functions $f: X \rightarrow \mathbb{R}$ on a topological space $X$. For a mes-measurable subset $X \subset \mathbb{R}$, by $L_{\text {loc }}^{1}(X)$ we denote the class of all locally mes-integrable functions with values in extended real axis $\mathbb{R}_{ \pm \infty}:=\{-\infty\} \cup \mathbb{R} \cup\{+\infty\}$, where $\mathbb{R}_{ \pm \infty}$ is equipped with the natural ordering $-\infty \leqslant x \leqslant+\infty, x \in \mathbb{R}_{ \pm \infty}$, and an ordering topology or topology of finite compactification on $\mathbb{R}$ with two ends $\pm \infty$. In the same way we define $L_{\text {loc }}^{1}(Y)$ for $Y \subset i \mathbb{R}$. 
Let $X \subset \mathbb{R}_{ \pm \infty}$. A function $f: X \rightarrow \mathbb{R}_{ \pm \infty}$ is increasing of for all $x_{1}, x_{2} \in X$, the inequality $x_{1} \leqslant x_{2}$ implies $f\left(x_{1}\right) \leqslant f\left(x_{2}\right)$; $f$ is decreasing if $-f$ is increasing.

An interval is a connected subset in $\mathbb{R}_{ \pm \infty}$. Stiltjes integrals over a bounded interval $I$ in $\mathbb{R}$ with end-points $a:=\inf I<\sup I=: b$ over functions of a bounded variation $m: \mathbb{R} \rightarrow \mathbb{R}$ on this interval $I$ is treated as

$$
\int_{a}^{b} \ldots \mathrm{d} m:=\int_{(a, b]} \ldots \mathrm{d} m, \quad I=(a, b], \quad-\infty<a<b<+\infty .
$$

if else is not said. Here $D(z, r):=\left\{z^{\prime} \in \mathbb{C}:\left|z^{\prime}-z\right|<r\right\}$ is an open circle, $\bar{D}(z, r):=\left\{z^{\prime} \in\right.$ $\left.\mathbb{C}:\left|z^{\prime}-z\right| \leqslant r\right\}$ is a closed circle, $\partial \bar{D}(z, r):=\bar{D}(z, r) \backslash D(z, r)$ is a circumference of radius $r \in \mathbb{R}^{+}$centered at $z \in \mathbb{C} ; D(r):=D(0, r), \bar{D}(r):=\bar{D}(0, r), \partial \bar{D}(r):=\partial \bar{D}(0, r)$. We define integrasl means over circumference $\partial \bar{D}(z, r)$ of a function $v: \partial \bar{D}(z, r) \rightarrow \mathbb{R}_{ \pm \infty}$ :

$$
\mathrm{C}_{v}(z, r):=: C(z, r ; v):=\frac{1}{2 \pi} \int_{0}^{2 \pi} v\left(z+r e^{i \theta}\right) \mathrm{d} \theta, \quad \mathrm{C}_{v}(r):=\mathrm{C}_{v}(0, r),
$$

over circle $D(z, r)$ for a function $v: D(z, r) \rightarrow \mathbb{R}_{ \pm \infty}$ :

$$
\mathrm{B}_{v}(z, r):=: B(z, r ; v):=\frac{2}{r^{2}} \int_{0}^{r} \mathrm{C}_{v}(z, t) t \mathrm{~d} t, \quad \mathrm{~B}_{v}(r):=\mathrm{B}_{v}(0, r),
$$

and also a supremum of a function $v: \partial \bar{D}(z, r) \rightarrow \mathbb{R}_{ \pm \infty}$ on circumference $\partial \bar{D}(z, r)$ :

$$
\mathrm{M}_{v}(z, r):=: M(z, r ; v):=\sup _{z^{\prime} \in \partial \bar{D}(z, r)} v\left(z^{\prime}\right), \quad \mathrm{M}_{v}(r):=\mathrm{M}_{v}(0, r),
$$

and as $v \in$ sbh, this coincides with $\sup _{\bar{D}(z, r)} v$. Of course, in 1.3 , for $1.3 \mathrm{C}$ ) and $1.3 \mathrm{~B}$ ) we assume the existence of all integrals, which always holds for the functions $v \in \mathrm{sbh}_{*}[2$, Def. 2.6.7, Thm.2.6.8], [1, 2.7] obeying

$$
\mathrm{B}_{v}(z, r) \leqslant \mathrm{C}_{v}(z, r) \leqslant \mathrm{M}_{v}(z, r) \quad \text { for all } \quad z \in \mathbb{C}, \quad r \in \mathbb{R}^{+} .
$$

In all results in [3]-12] mentioned above in Section 1.1, two objects played a key role. First, these are special integrals over intervals on real or imaginary axis, often called logarithmic integrals.

Definition 1. For a function $v \in L_{\text {loc }}^{1}(\mathbb{R})$ we let

$$
J_{\mathbb{R}}(r, R ; v):=\frac{1}{2 \pi} \int_{r}^{R} \frac{v(x)+v(-x)}{x^{2}} \mathrm{~d} x, \quad 0<r<R<+\infty,
$$

and for a function $v \in L_{\mathrm{loc}}^{1}(i \mathbb{R})$ we let

$$
J_{i \mathbb{R}}(r, R ; v):=\frac{1}{2 \pi} \int_{r}^{R} \frac{v(-i y)+v(i y)}{y^{2}} \mathrm{~d} y, \quad 0<r<R<+\infty .
$$

The second object is logarithmic measures of the intervals for the sequences of points $\mathbf{Z}=$ $\left\{\mathbf{z}_{k}\right\}_{k=1,2, \ldots} \subset \mathbb{C}$. We identify each sequence $\mathbf{Z}$ with a counting measure $n_{\mathbf{Z}} \in$ Meas $^{+}$defined as

$$
n_{\mathrm{Z}}(S):=\sum_{\mathrm{z}_{k} \in S} 1 \text { for all } S \subset \mathbb{C},
$$

and we define these logarithmic measures for an arbitrary measure in Meas $^{+}$. 
Definition 2. Given a measure $\mu \in$ Meas $^{+}$,

$$
\begin{aligned}
l_{\mu}^{\mathrm{rh}}(r, R) & :=\int_{\substack{r<|z| \leqslant R \\
\operatorname{Re} z>0}} \operatorname{Re} \frac{1}{z} \mathrm{~d} \mu(z), \quad 0<r<R<+\infty, \\
l_{\mu}^{\mathrm{lh}}(r, R):= & \int_{\substack{r<|z| \leqslant R \\
\operatorname{Re} z<0}} \operatorname{Re}\left(-\frac{1}{z}\right) \mathrm{d} \mu(z), \quad 0<r<R<+\infty,
\end{aligned}
$$

are a right and left logarithmic measures of intervals $(r, R] \subset \mathbb{R}^{+}$for the measure $\mu$ respectively. They generate a logarithmic submeasure of intervals

$$
l_{\mu}(r, R):=\max \left\{l_{\mu}^{\mathrm{lh}}(r, R), l_{\mu}^{\mathrm{rh}}(r, R)\right\}, \quad 0<r<R<+\infty .
$$

\subsection{Main result.}

Theorem 1. Assume that functions

$$
\begin{aligned}
& M \in \mathrm{sbh}_{*}, \quad \text { type }[M] \stackrel{\mid 1.1}{<}+\infty, \quad \mu:=\mu_{M}:=\frac{1}{2 \pi} \Delta M \in \mathrm{Meas}^{+}, \\
& u \in \mathrm{sbh}_{*}, \quad \text { type }[u]<+\infty, \quad \nu:=\nu_{u}:=\frac{1}{2 \pi} \Delta u \in \mathrm{Meas}^{+}, \\
& q_{0}: \mathbb{R} \rightarrow \mathbb{R}^{+} \cup\{+\infty\}, \quad q_{0} \in L_{\mathrm{loc}}^{1}(\mathbb{R}), \\
& q: \mathbb{R} \rightarrow \mathbb{R}^{+}, \quad q \in C(\mathbb{R}), \quad \limsup _{|y| \rightarrow+\infty} \frac{q(y)}{|y|}<1,
\end{aligned}
$$

and some mes-measurable subset $E \subset \mathbb{R}^{+}$with

$$
E^{r}:=E \cap[0, r], \quad q_{E}(r):=\operatorname{mes}\left(E^{r}\right) \ln \frac{e r}{\operatorname{mes}\left(E^{r}\right)}=: q_{E}(-r),
$$

the inequalities hold:

$$
\begin{aligned}
u(i y)+u(-i y) & \leqslant \mathrm{C}_{M}(i y, q(y))+\mathrm{C}_{M}(-i y, q(-y)) \\
& +q_{0}(y)+q_{0}(-y) \text { for each } y \in \mathbb{R}^{+} \backslash E .
\end{aligned}
$$

Then for all numbers $r_{0}>0$ and $N \in \mathbb{R}^{+}$there exists a number $C \in \mathbb{R}^{+}$, for which

$$
\begin{aligned}
\max \left\{l_{\nu}(r, R), J_{i \mathbb{R}}(r, R ; u)\right\} \leqslant & \min \left\{l_{\mu}^{\mathrm{rh}}(r, R), l_{\mu}^{\mathrm{lh}}(r, R), J_{i \mathbb{R}}(r, R ; M)\right\} \\
& +C J_{\mathbb{R}}\left(r, R ; q_{0}+q_{E}\right)+C I_{N}(r, R ; q)+C
\end{aligned}
$$

for all $r_{0} \leqslant r<R<+\infty$, where

$$
I_{N}(r, R ; q):=\int_{r}^{R} t^{N} \sup _{s \geqslant t} \frac{q(s)+q(-s)}{s^{2+N}} \mathrm{~d} t .
$$

Remark 1. For the functions in $1.8 \mathrm{M})$ and $1.8 \mathrm{u})$, their Riesz measures $\mu$ and $\nu$ are of a finite type or of a finite upper density with order 1, which means that

$$
\text { type }[\mu]:=\limsup _{r \rightarrow+\infty} \frac{\mu^{\mathrm{rad}}(r)}{r}<+\infty, \quad \mu^{\mathrm{rad}}(r):=\mu(D(0, r)), \quad \text { type }[\nu]<+\infty .
$$

Remark 2. By inequalities (1.4), the means over circumferences $\mathrm{C}_{M}$ in $\left.1.3 \mathrm{C}\right)$ in the right hand side of inequality $(1.9 \mathrm{C})$ can be replaced by the means over the circumferences $\mathrm{B}_{M}$ in $\left(1.3 \mathrm{~B}\right.$, but they can not be replaced by the suprema over the circumferences or circles $\mathrm{M}_{M}$ in (1.3M). 


\section{Proof of TheOREM 1}

In the proof of Theorem 1 we can consider arbitrary but fixed value $r_{0}>0$ since according Definition 2 of logarithmic measures of intervals $(1.7 \mathrm{r}),(1.7 \mathrm{l}),(1.7 \mathrm{~m})$ and Definition 1 of integrals 1.5 , 1.5 , by changing $r_{0}>0$ in conclusion 1.10) of Theorem 1, one just increases the constant $C \in \mathbb{R}^{+}$. This is why, in the proof of Theorem 1 , we shall increase the value $r_{0}$ if needed not saying this explicitly.

If in condition (1.8 a) we consider the function $q+1$ instead of $q$, its properties in (1.8 a remain the same as well as inequality $(1.9 \mathrm{C})$ and this makes no influence on conclusion $(1.10)$. This is why we can suppose that $q \geqslant 1$ on $\mathbb{R}$. We consider a regular domain for the Dirichlet problem [1, Thm. 2.11]:

$$
D:=D_{q}:=\{z \in \mathbb{C}:-q(\operatorname{Im} z)<\operatorname{Re} z<q(\operatorname{Im} z)\} .
$$

For $b \in \mathbb{R}^{+}$we make use of the notation:

$$
\operatorname{str}_{b}:=\{z \in \mathbb{C}:|\operatorname{Im} z|<b\}, \quad \overline{\operatorname{str}}_{b}:=\{z \in \mathbb{C}:|\operatorname{Im} z| \leqslant b\}
$$

respectively for open and closed strips of width $2 b$ with the reference line $\mathbb{R}$. By the limiting condition in (1.8p), for sufficiently large $b>0$, a part $D \backslash \overline{\operatorname{str}}_{b}$ of the domain $D$ consists of two simply-connected domains located inside some pair of open vertical angles of opening at most $\pi$ :

$$
\angle(\alpha, \pi-\alpha):=\{z \in \mathbb{C}: \alpha<\arg z<\pi-\alpha\}, \quad \angle(-\pi+\alpha,-\alpha) \text { with } \alpha \in(0, \pi / 2) .
$$

We can make a classical balayage of a function $M \in \mathrm{sbh}_{*}$ from the domain $D$ in two steps. First we make balayage from this pair of simply-connected domains and a balayaged function becomes a subharmonic function of a finite type since it does not exceed a classical balayage of kind 0 of the function $M$ from the pair of vertical angles (2.3) [13, 6.2, Thms. 7, 8]. At the second step we make a classical balayage from $D$ for a logarithmic potential of kind 0 of the arisen measure with a compact support in the closure $D \cap \overline{\operatorname{str}}_{b}$ [14, Ch. IV, Sect. 1], [15, Thm. 2.5.3.1], which can increase the growth of the logarithmic potential at most by a quantity of order $O(\ln |z|), z \rightarrow \infty$. Thus, such construction gives a subharmonic function

$$
M^{D}=\left\{\begin{array}{l}
M \quad \text { on the complement } \mathbb{C} \backslash D, \\
\text { harmonic continuation of } M \text { inside } D \text { on } D
\end{array}\right.
$$

of a finite type, that is, with type $\left[M^{D}\right]<+\infty$. At that, by the maximum principle,

$$
\mathrm{C}_{M}(i y, q(y)) \leqslant M^{D}(i y) \text { for all } y \in \mathbb{R}, \quad M \leqslant M^{D} \text { on } \mathbb{C},
$$

and it follows from inequalities $(1.9 \mathrm{C})$ that

$$
u(i y)+u(-i y) \leqslant M^{D}(i y)+M^{D}(-i y)+q_{0}(y)+q_{0}(-y) \text { for all } y \in \mathbb{R} \backslash E .
$$

For a measure $\nu \in$ Meas $^{+}$, the restriction of the measure $\nu$ on $S \subset \mathbb{C}$ is denoted as $\left.\nu\right|_{S}$.

The Riesz measure $\frac{1}{2 \pi} \Delta M^{D}$ of the function $M^{D}$ is a sum of its restrictions

$$
\mu_{\infty}:=\left.\frac{1}{2 \pi} \Delta M^{D}\right|_{\mathbb{C} \backslash \operatorname{clos} D}=\left.\mu\right|_{\mathbb{C} \backslash \operatorname{clos} D} \leqslant \mu, \quad \mu_{0}:=\left.\frac{1}{2 \pi} \Delta M^{D}\right|_{\partial D},
$$

where clos $D$ and $\partial D$ are respectively closure and boundary of $D$. Integrating inequality (2.5) with the factor $\frac{1}{2 \pi}$ in the notation

$$
E_{r}^{R}:=E^{R} \backslash E^{r}=E \cap(r, R]
$$


for all $r_{0} \leqslant r<R<+\infty$ gives the inequalities

$$
\begin{aligned}
J_{i \mathbb{R}}(r, R ; u) \leqslant & J_{i \mathbb{R}}\left(r, R ; M^{D}\right) \\
& +\frac{1}{2 \pi} \int_{E_{r}^{R}} \frac{u(i y)+u(-i y)-M^{D}(i y)-M^{D}(-i y)}{y^{2}} \mathrm{~d} y+J_{\mathbb{R}}\left(r, R ; q_{0}\right) .
\end{aligned}
$$

Lemma 1. Let $r_{0}>0$. For each function $u$ in $1.8 \mathrm{u}$ there exists a number $c_{u} \in \mathbb{R}^{+}$such that for each mes-measurable subset $E \subset \mathbb{R}^{+}$, in terms of notations $(1.9 \mathrm{E}$ ) and (2.7), the inequality holds:

$$
\int_{E_{r}^{R}} \frac{|u|(x)}{x^{2}} \mathrm{~d} x \leqslant c_{u} \int_{r}^{R} \frac{q_{E}(t)}{t^{2}} \mathrm{~d} t+c_{u} \quad \text { for all } \quad r_{0} \leqslant r<R<+\infty,
$$

where the function $q_{E}$ is increasing and $q_{E}(r) \leqslant r$ as $r \in \mathbb{R}_{*}^{+}$.

Proof. The function

$$
(x, y) \longmapsto x \ln \frac{e y}{x}, \quad(x, y) \in(0, y] \times \mathbb{R}^{+},
$$

redefined by zero at $x=0$, increases in the variable $y \in \mathbb{R}_{*}^{+}$, as well as in $x \in(0, y]$, attaining its maximal value as $x=y$ and this gives the properties of the function $q_{E}$. In what follows, the symbol $\mathbf{1}_{E}$ stands for the characteristic function of the subset $E$.

According [16, Thm. 8], there exist constants $c^{\prime}, c \in \mathbb{R}^{+}$obeying

$$
\int_{r_{0}}^{x} \mathbf{1}_{E}(t)|u|(t) \mathrm{d} t \leqslant c^{\prime} x \operatorname{mes}\left(E^{x}\right) \ln \frac{4 x}{\operatorname{mes}\left(E^{x}\right)} \stackrel{1.9 \mathrm{E}}{\leqslant} c q_{E}(x) x
$$

for all $x \geqslant r_{0}$. For the left hand side in $(2.9)$ we have

$$
\begin{aligned}
\int_{E_{r}^{R}} \frac{|u|(x)}{x^{2}} \mathrm{~d} x & =\int_{r}^{R} \mathbf{1}_{E}(x) \frac{|u|(x)}{x^{2}} \mathrm{~d} x=\int_{r}^{R} \frac{1}{x^{2}} \mathrm{~d} \int_{r}^{x} \mathbf{1}_{E}(t)|u|(t) \mathrm{d} t \mathrm{~d} x \\
& =\frac{1}{R^{2}} \int_{r}^{R} \mathbf{1}_{E}(t)|u|(t) \mathrm{d} t+\int_{r}^{R} \int_{r}^{x} \mathbf{1}_{E}(t)|u|(t) \mathrm{d} t \mathrm{~d}\left(-\frac{1}{x^{2}}\right) \\
& \leqslant c \frac{q_{E}(R)}{R}+2 c \int_{r}^{R} \frac{q_{E}(x)}{x^{2}} \mathrm{~d} x \leqslant c+2 c \int_{r}^{R} \frac{q_{E}(x)}{x^{2}} \mathrm{~d} x,
\end{aligned}
$$

since the inequality $q_{E}(R) \leqslant R$ holds. The proof is complete.

Applying four times Lemma 1 to the integral over the set $E_{r}^{R}$ in the right hand side 2.8), for some number $c_{1} \in \mathbb{R}^{+}$and all $r_{0} \leqslant r<R<+\infty$ we obtain

$$
J_{i \mathbb{R}}(r, R ; u) \leqslant J_{i \mathbb{R}}\left(r, R ; M^{D}\right)+c_{1} J_{\mathbb{R}}\left(r, R ; q_{E}+q_{0}\right)+c_{1} .
$$

Lemma 2 ([17, Prop. 4.1, (4.19)]). Let $r_{0}>0$. For each function $u$ in $(1.8 \mathrm{a})$ there exists a number $c_{u} \in \mathbb{R}^{+}$such that

$$
\max \left\{\left|J_{i \mathbb{R}}(r, R ; u)-l_{\nu}^{\mathrm{rh}}(r, R)\right|,\left|J_{i \mathbb{R}}(r, R ; u)-l_{\nu}^{\mathrm{lh}}(r, R)\right|\right\} \leqslant c_{u}
$$

for all $r_{0} \leqslant r<R<+\infty$. 
We apply twice Lemma 2 to the functions $u$ and $M^{D}$ in (2.11) and for some number $c_{2} \in \mathbb{R}^{+}$ and all $r_{0} \leqslant r<R<+\infty$ we have the inequalities

$$
\begin{aligned}
l_{\nu}^{\mathrm{rh}}(r, R) & \stackrel{2.6}{\leqslant} l_{\mu_{\infty}+\mu_{0}}^{\mathrm{rh}}(r, R)+c_{1} J_{\mathbb{R}}\left(r, R ; q_{E}+q\right)+c_{2} \\
& \stackrel{2.6}{\leqslant} l_{\mu}^{\mathrm{rh}}(r, R)+l_{\mu_{0}}^{\mathrm{rh}}(r, R)+c_{1} J_{\mathbb{R}}\left(r, R ; q_{E}+q_{0}\right)+c_{2} .
\end{aligned}
$$

Assume that the angles are chosen and we fix a number $b>0$ as in (2.1)-(2.3). The restriction of the measure $\mu_{0}$ on a closed strip $\overline{\operatorname{str}}_{b}$ is a measure with a compact support and for this measure the logarithmic measures and submeasures of the intervals in (1.7) are uniformly bounded for all $r_{0} \leqslant r<R<+\infty$. Therefore, without loss of generality, we can assume that the support of the measure $\mu_{0}$ is contained in the pair of angles $(2.3)$ and is disjoint with the open strip $\operatorname{str}_{b}$. We denote the $\mu_{0}$-measure of the closed strip $\overline{\operatorname{str}}_{y}$ of width $2 y \in \mathbb{R}^{+}$by

$$
\mu_{0}^{i}(y):=\mu_{0}\left(\overline{\operatorname{str}}_{y}\right), \quad \text { where } \quad \mu_{0}^{i}(y) \leqslant C y \quad \text { for all } y \in \mathbb{R}^{+}
$$

for some constant $C$ independent of $y$ and also $\mu_{0}^{i}(y)=0$ as $y \in[0, b)$.

Lemma 3. Let $r_{0}>0$ and $\mu_{0} \in$ Meas $^{+}$be a measure with a support supp $\mu_{0}$ in the closure $D \stackrel{(2.1)}{=} D_{q}$, where the function $q$ is from (1.8q) and the function $\mu_{0}^{i}: \mathbb{R}^{+} \rightarrow \mathbb{R}^{+}$is from (2.14). Then for some number $C_{0} \in \mathbb{R}^{+}$the inequalities hold:

$$
l_{\mu_{0}}^{\mathrm{rh}}(r, R) \leqslant \int_{r}^{R} \frac{Q(y)}{y^{2}} \mathrm{~d} \mu_{0}^{i}(y)+C_{0} \quad \text { for all } \quad r_{0} \leqslant r<R<+\infty,
$$

where

$$
Q(y):=q(y)+q(-y), \quad y \in \mathbb{R}^{+} .
$$

Proof. For all $b \leqslant r<R<+\infty$ we have

$$
\begin{aligned}
& l_{\mu_{0}}^{\mathrm{rh}}(r, R) \stackrel{\text { 1.7月 }}{=} \int_{\substack{r<|z| \leqslant R \\
\operatorname{Re} z>0}} \frac{\operatorname{Re} z}{|z|^{2}} \mathrm{~d} \mu_{0}(z) \stackrel{2.1}{\leqslant} \int_{\substack{r<|z| \leqslant R \\
\operatorname{Re} z>0}} \frac{q(\operatorname{Im} z)}{|\operatorname{Im} z|^{2}} \mathrm{~d} \mu_{0}(z) \\
& \stackrel{2.14}{\leqslant} \int_{r \sin \alpha}^{R} \frac{q(y)+q(-y)}{y^{2}} \mathrm{~d} \mu_{0}^{i}(y) \stackrel{3.1}{\leqslant}\left(\int_{r \sin \alpha}^{r}+\int_{r}^{R}\right) \frac{Q(y)}{y^{2}} \mathrm{~d} \mu_{0}^{i}(y) \\
& \stackrel{2.14}{\leqslant} C \frac{Q(r)}{r \sin ^{2} \alpha}+\int_{r}^{R} \frac{Q(y)}{y^{2}} \mathrm{~d} \mu_{0}^{i}(y) \stackrel{1.8 \mathrm{~A}}{\leqslant} C_{0}+\int_{r}^{R} \frac{Q(y)}{y^{2}} \mathrm{~d} \mu_{0}^{i}(y),
\end{aligned}
$$

where $C_{0} \in \mathbb{R}^{+}$is independent of $b \leqslant r<R<+\infty$.

By Lemma 3 and inequality 2.13 with a constant $C_{1} \in \mathbb{R}^{+}$we obtain

$$
l_{\nu}^{\mathrm{rh}}(r, R) \stackrel{2.6}{\leqslant} l_{\mu}^{\mathrm{rh}}(r, R)+\int_{r}^{R} \frac{Q(y)}{y^{2}} \mathrm{~d} m(y)+c_{1} J_{\mathbb{R}}\left(r, R ; q_{E}+q_{0}\right)+C_{1},
$$

where we let $m(t):=\mu_{0}^{i}(t)$. By construction of $m$, there exists a constant $C \in \mathbb{R}^{+}$, for which the inequalities hold:

$$
m(t) \stackrel{2.14}{\leqslant} C t, \quad \text { for all } t \in \mathbb{R}^{+}, \quad m(t)=0 \quad \text { as } \quad t \in[0, b) \neq \varnothing .
$$


Lemma 4. Let an increasing function $m: \mathbb{R}^{+} \rightarrow \mathbb{R}^{+}$satisfies conditions (2.19), and a continuous function

$$
Q: \mathbb{R}^{+} \rightarrow \mathbb{R}^{+} \quad \text { is such that } Q(t)=O(t) \quad \text { as } t \rightarrow+\infty .
$$

Then for each number $N \in \mathbb{R}^{+}$there exists a number $C_{2} \in \mathbb{R}^{+}$obeying

$$
\int_{r}^{R} \frac{Q(t)}{t^{2}} \mathrm{~d} m(t) \leqslant C_{2} \int_{r}^{R} t^{N} \sup _{s \geqslant t} \frac{Q(s)}{s^{2+N}} \mathrm{~d} t+C_{2}
$$

for all $b \leqslant r<R<+\infty$,

Proof. For the integral in the left hand side in 2.21) we have

$$
I:=\int_{r}^{R} \frac{Q(t)}{t^{2}} \mathrm{~d} m(t)=\int_{r}^{R} \frac{Q(t)}{t^{2} t^{N}} \mathrm{~d} \int_{r}^{t} s^{N} m(s) \leqslant \int_{r}^{R} \sup _{s \geqslant t} \frac{Q(s)}{s^{2+N}} \mathrm{~d} \int_{r}^{t} s^{N} m(s) .
$$

Thanks to 2.20), for the integrand in the latter integral being decreasing function there exists a number $C_{3}$, for which

$$
T_{N}(t):=\sup _{s \geqslant t} \frac{Q(s)}{s^{2+N}} \leqslant C_{3} \sup _{s \geqslant t} \frac{s}{s^{2+N}}=C_{3} t^{-N-1} \quad \text { for all } \quad t \in[b,+\infty) .
$$

Integrating this integral by parts, we get:

$$
I \leqslant T_{N}(R) \int_{r}^{R} s^{N} \mathrm{~d} m(s)+\int_{r}^{R} \int_{r}^{t} s^{N} \mathrm{~d} m(s) \mathrm{d}\left(-T_{N}(t)\right) .
$$

In view of (2.19), we can estimate the integral:

$$
\int_{r}^{t} s^{N} \mathrm{~d} m(s) \leqslant m(t) t^{N} \leqslant C t^{N+1}
$$

and in view of 2.22 , for the right hand side in 2.23) this gives:

$$
I \leqslant C C_{3}+C \int_{r}^{R} t^{N+1} \mathrm{~d}\left(-T_{N}(t)\right) \leqslant C C_{3}+C C_{3}+C(N+1) \int_{r}^{R} T_{N}(t) t^{N} \mathrm{~d} t .
$$

For $C_{2}:=\max \left\{2 C C_{3}, C(N+1)\right\}$ this implies exactly (2.21). The proof is complete.

By 2.18 and inequality 2.21) in Lemma 4 , for some constant $C_{4} \in \mathbb{R}^{+}$we get

$$
l_{\nu}^{\mathrm{rh}}(r, R) \stackrel{2.6}{\leqslant} l_{\mu}^{\mathrm{rh}}(r, R)+C_{4} \int_{r}^{R} t^{N} \sup _{s \geqslant t} \frac{Q(s)}{s^{2+N}} \mathrm{~d} t+c_{1} J_{\mathbb{R}}\left(r, R ; q_{E}+q_{0}\right)+C_{1}
$$

for all $+\infty>R>r \geqslant \max \left\{r_{0}, b\right\}$, where the quantity $\max \left\{r_{0}, b\right\}$ can be replaced by $r_{0}$ increasing if needed the constant $C_{1}$, and $Q$ is a function from (2.16).

Lemma 5. Let $r_{0}>0$. For each function $u$ in $(1.8 \mathrm{u})$ there exists $C_{u} \in \mathbb{R}^{+}$such that for all $r_{0} \leqslant r<R<+\infty$ we have the inequality

$$
\max \left\{l_{\nu}(r, R), J_{i \mathbb{R}}(r, R ; u)\right\} \leqslant \min \left\{l_{\nu}^{\mathrm{rh}}(r, R), l_{\nu}^{\mathrm{lh}}(r, R), J_{i \mathbb{R}}(r, R ; u)\right\}+C_{u} .
$$

The statement of this lemma follows immediately lemma 2.

Applying Lemma 5 to the functions $u$ and $M$ with the Riesz measures $\nu$ and $\mu$, by (2.24) we obtain 1.10 and this completes the proof of Theorem 1 . 


\section{VARIATIONS OF CONCLUSION (1.10) OF THEOREM 1}

3.1. Some simplifications. Rather cumbersome integrals $(1.11)$ involved in the right hand side of the final estimate (1.10) in Theorem 1 can be included into our standard logarithmic integral $J_{\mathbb{R}}$ of form $(1.5 \mathrm{R})$ already involved in the right hand side in the estimate 1.10 . This can be done under inessential additional restrictions for the function

$$
Q_{N}(s):=\frac{Q(s)}{s^{2+N}}, \quad Q(s) \stackrel{2.16}{=} q(s)+q(-s), \quad s \in \mathbb{R}^{+} .
$$

Proposition 1. Assume that there exists an interval $(A,+\infty) \neq \varnothing$, on which at least one of the following two conditions hold:

(i) the function $Q_{N}$ in (3.1) is decreasing in $(A,+\infty)$;

(ii) the function $Q$ in (2.16) is continuously differentiable on $(A,+\infty)$ and possesses the property

$$
\limsup _{y \rightarrow+\infty} \frac{y Q^{\prime}(y)}{Q(y)}<+\infty .
$$

Then there exist the numbers $r_{0}>0, N \in \mathbb{R}^{+}$and $C \in \mathbb{R}^{+}$, for which

$$
I_{N}(r, R ; q) \stackrel{1.11}{=} \int_{r}^{R} t^{N} \sup _{s \geqslant t} Q_{N}(s) \mathrm{d} t \leqslant C \int_{r}^{R} \frac{Q(t)}{t^{2}} \mathrm{~d} t \stackrel{(1.5)}{=} C J_{\mathbb{R}}(r, R ; q) .
$$

In particular, the latter integral in 1.10 can be replaced by $J_{\mathbb{R}}(r, R ; q)$, and the final estimate 1.10 in Theorem 1 can be written as

$$
\begin{array}{r}
\max \left\{l_{\nu}(r, R), J_{i \mathbb{R}}(r, R ; u)\right\} \leqslant \min \left\{l_{\mu}^{\mathrm{rh}}(r, R), l_{\mu}^{\mathrm{lh}}(r, R), J_{i \mathbb{R}}(r, R ; M)\right\} \\
+C J_{\mathbb{R}}\left(r, R ; q_{0}+q_{E}+q\right)+C \text { for all } r_{0} \leqslant r<R<+\infty,
\end{array}
$$

Proof. If the function (3.1) is decreasing on $(A,+\infty)$, then it is obvious that

$$
t^{N} \sup _{s \geqslant t} Q_{N}(s) \stackrel{(3.1)}{=} t^{N} \frac{Q(t)}{t^{2+N}}=\frac{Q(t)}{t^{2}}
$$

for all $t>A$ and for $r_{0}>A$ we get exactly (3.3).

For a continuously differentiable function $Q$ satisfying condition $(3.2)$ there exists $C_{4} \in \mathbb{R}^{+}$ such that $s Q^{\prime}(s) \leqslant C_{5} Q(s)$ for all $s \geqslant b$. The derivative of the function in (3.1) reads as

$$
Q_{N}^{\prime}(s)=\frac{Q^{\prime}(s) s-(2+N) Q(s)}{s^{3+N}} \leqslant \frac{C_{5} Q(s)-(2+N) Q(s)}{s^{3+N}}
$$

and it is negative for $N \geqslant C_{5}-2$. This means that the function $Q_{N}$ in 3.1 is decreasing.

Proposition 1 implies immediately a following obvious corollary.

Corollary 1. If under conditions (ii) and (ii) of Proposition 1 for some number $r_{0}>0$ the relation holds:

$$
\sup _{r_{0} \leqslant r<R<+\infty} J_{\mathbb{R}}\left(r, R ; q_{0}+q_{E}+q\right)<+\infty,
$$

then the final estimate 1.10 in Theorem 1 can be written as

$$
\max \left\{l_{\nu}(r, R), J_{i \mathbb{R}}(r, R ; u)\right\} \stackrel{\sqrt[3.4]{5}}{\leqslant} \min \left\{l_{\mu}^{\mathrm{rh}}(r, R), l_{\mu}^{\mathrm{lh}}(r, R), J_{i \mathbb{R}}(r, R ; M)\right\}+C
$$

for all $r_{0} \leqslant r<R<+\infty$.

By a known asymptotics of the functions $q_{0}, q_{E}, q$ at $\pm \infty$, we can also simplify the final estimate 1.10 of Theorem 1. One of the versions is provided in the following proposition. 
Proposition 2. Let a function $P: \mathbb{R}^{+} \rightarrow \mathbb{R}^{+}$be bounded and integrable in the Riemann sense on each bounded interval $I \subset \mathbb{R}^{+}$satisfy the condition

$$
\limsup _{t \rightarrow+\infty} \frac{P(t)}{t}=0
$$

Then for each number $r_{0}>0$ there exists a decreasing function $d: \mathbb{R}^{+} \rightarrow \mathbb{R}^{+}$such that

$$
\lim _{R \rightarrow+\infty} d(R)=0, \quad \int_{r}^{R} \frac{P(t)}{t^{2}} \mathrm{~d} t \leqslant d(R) \ln \frac{R}{r} \quad \text { for all } \quad r_{0} \leqslant r<R<+\infty .
$$

In particular, if the functions $q_{0}, q, q_{E}$ in $\left.11.8 \mathrm{p}, 1.8 \mathrm{p}\right), 1.9 \mathrm{E}$ ) satisfy the conditions

$$
\limsup _{|y| \rightarrow+\infty} \frac{q_{0}(y)+q(y)+q_{E}(y)}{|y|}=0, \quad \sup _{[-R, R]} q_{0}<+\infty \quad \text { for all } R \in \mathbb{R}^{+}
$$

and the function $q_{0}$ is locally integrable in the Riemann sense, then final estimate (1.10) in Theorem 1 can be written as

$$
\begin{aligned}
\max \left\{l_{\nu}(r, R), J_{i \mathbb{R}}(r, R ; u)\right\} \stackrel{\sqrt[3.4]{\leqslant}}{\leqslant} & \min \left\{l_{\mu}^{\mathrm{rh}}(r, R), l_{\mu}^{\mathrm{hh}}(r, R), J_{i \mathbb{R}}(r, R ; M)\right\} \\
& +d(R) \ln \frac{R}{r}+C \quad \text { for all } \quad r_{0} \leqslant r<R<+\infty
\end{aligned}
$$

where $d$ is a decreasing function such that $d(R)=o(1)$ as $R \rightarrow+\infty$.

Proof. We first pass to the function

$$
p(t):=\sup _{s \geqslant t} \frac{P(s)}{s} \geqslant \frac{P(t)}{t}, \quad t \in \mathbb{R}^{+} ; \quad \int_{r}^{R} \frac{p(t)}{t} \mathrm{~d} t \geqslant \int_{r}^{R} \frac{P(t)}{t^{2}} \mathrm{~d} t .
$$

decaying in view of $(3.5)$. For each fixed number $R>0$ we let

$$
\begin{aligned}
d(R) & :=\sup _{r_{0} \leqslant r<R} \frac{1}{\ln (R / r)} \int_{r}^{R} \frac{p(t)}{t} \mathrm{~d} t \geqslant \frac{1}{\ln (R / r)} \int_{r}^{R} \frac{p(t)}{t} \mathrm{~d} t \\
& \geqslant \frac{1}{\ln (R / r)} \int_{r}^{R} \frac{P(t)}{t^{2}} \mathrm{~d} t \text { for all } r_{0} \leqslant r<R<+\infty,
\end{aligned}
$$

which gives the latter inequality in (3.6).

The supremum in (3.8) is taken for the function with partial derivatives

$$
\begin{aligned}
& \frac{\partial}{\partial r} \frac{1}{\ln (R / r)} \int_{r}^{R} \frac{p(t)}{t} \mathrm{~d} t=\frac{1}{r \ln ^{2}(R / r)} \int_{r}^{R} \frac{p(t)-p(r)}{t} \mathrm{~d} t \leqslant 0 \quad \text { as } \quad r_{0} \leqslant r<R, \\
& \frac{\partial}{\partial R} \frac{1}{\ln (R / r)} \int_{r}^{R} \frac{p(t)}{t} \mathrm{~d} t=\frac{1}{R \ln ^{2}(R / r)} \int_{r}^{R} \frac{p(R)-p(t)}{t} \mathrm{~d} t \leqslant 0 \quad \text { as } \quad r_{0} \leqslant r<R,
\end{aligned}
$$

and hence, this functions decreases in $r<R$. Therefore,

$$
d(R) \equiv \frac{1}{\ln \left(R / r_{0}\right)} \int_{r_{0}}^{R} \frac{p(t)}{t} \mathrm{~d} t
$$

is a decreasing function on $\left[r_{0},+\infty\right)$. 
We choose a number $a>0$ and let $p(t) \leqslant a$ as $t \geqslant R_{a}$. Then by $(3.9)$, for $R>R_{a} \geqslant r_{0}$ we have

$$
d(R)=\frac{1}{\ln \left(R / r_{0}\right)}\left(\int_{r_{0}}^{R_{a}}+\int_{R_{a}}^{R}\right) \frac{p(t)}{t} \mathrm{~d} t \leqslant \frac{\left(\sup _{\left[r_{0}, R_{a}\right]} p\right) \ln \left(R_{a} / r_{0}\right)}{\ln \left(R / r_{0}\right)}+a \frac{\ln \left(R / R_{a}\right)}{\ln \left(R / r_{0}\right)}
$$

Hence, $\lim \sup _{R \rightarrow+\infty} d(R) \leqslant a$, and by the arbitrary choice of the number $a>0$ this leads us to the relation $d(R)=o(1)$ as $R \rightarrow+\infty$.

3.2. Logarithmic measures and submeasures of intervals. The notions of logarithmic measures of intervals $1.7 \mathrm{c}),(1.7 \mathrm{l})$ and logarithmic submeasures of intervals $(1.7 \mathrm{~m})$ in Definition 2 can be introduced in another form.

Definition 3. Let $\mu \in$ Meas $^{+}$. We introduce a counting function of the measure $\mu$ with a $2 \pi$-periodic Borel positive weight function $k: \mathbb{R} \rightarrow \mathbb{R}^{+}$:

$$
\mu(r ; k):=\int_{\bar{D}(r)} k(\arg z) \mathrm{d} \mu(z),
$$

As $k \equiv 1$, we obviously have $\mu(r ; 1) \stackrel{1.12}{\equiv} \mu^{\mathrm{rad}}(r)$ for $r \in \mathbb{R}^{+}$.

In the particular cases $k=\cos ^{ \pm}$, that is,

$$
k(\theta):=\cos ^{+} \theta:=\max \{0, \cos \theta\}, \quad k(\theta):=\cos ^{-} \theta:=\max \{0,-\cos \theta\}, \quad \theta \in \mathbb{R},
$$

by Definitions (1.7), in terms of notations (3.10)-(3.11), we integrate by parts for $0<r<R<$ $+\infty$ :

$$
\begin{aligned}
& l_{\mu}^{\mathrm{rh}}(r, R) \stackrel{1.7 \mathrm{f}}{=} \int_{r}^{R} \frac{\mathrm{d} \mu\left(t ; \cos ^{+}\right)}{t} \\
& =\frac{\mu\left(R ; \cos ^{+}\right)}{R}-\frac{\mu\left(r ; \cos ^{+}\right)}{r}+\int_{r}^{R} \frac{\mu\left(t ; \cos ^{+}\right)}{t^{2}} \mathrm{~d} t, \\
& l_{\mu}^{\mathrm{lh}}(r, R) \stackrel{\text { 1.7] }}{=} \int_{r}^{R} \frac{1}{t} \mathrm{~d} \mu\left(t ; \cos ^{-}\right) \\
& =\frac{\mu\left(R ; \cos ^{-}\right)}{R}-\frac{\mu\left(r ; \cos ^{-}\right)}{r}+\int_{r}^{R} \frac{\mu\left(t ; \cos ^{-}\right)}{t^{2}} \mathrm{~d} t .
\end{aligned}
$$

We let:

$$
\begin{aligned}
& \breve{l}_{\mu}^{\mathrm{rh}}(r, R) \stackrel{3.12 \mathrm{~s}}{:=} \int_{r}^{R} \frac{\mu\left(t ; \cos ^{+}\right)}{t^{2}} \mathrm{~d} t, \quad 0<r<R<+\infty \\
& \breve{l}_{\mu}^{\mathrm{lh}}(r, R) \stackrel{3.12 \mathrm{l}}{:=} \int_{r}^{R} \frac{\mu\left(t ; \cos ^{-}\right)}{t^{2}} \mathrm{~d} t, \quad 0<r<R<+\infty, \\
& \breve{l}_{\mu}(r, R) \stackrel{1.7 \mathrm{~m}}{:=} \max \left\{\breve{l}_{\mu}^{\mathrm{h}}(r, R), \breve{l}_{\mu}^{\mathrm{rh}}(r, R)\right\}, \quad 0<r<R<+\infty \text {. }
\end{aligned}
$$


Proposition 3. Let $\mu \in$ Meas $^{+}$be a measure of an upper finite density or of a finite type type $[\mu] \stackrel{\frac{1.12}{<}}{<}+\infty$ and $r_{0}>0$. Then

$$
\left\{\begin{array}{l}
\left|\breve{l}_{\mu}^{\mathrm{rh}}(r, R)-l_{\mu}^{\mathrm{rh}}(r, R)\right|=O(1) \\
\left|\breve{l}_{\mu}^{\mathrm{h}}(r, R)-l_{\mu}^{\mathrm{h}}(r, R)\right|=O(1) \quad \text { for all } \quad r_{0} \leqslant r<R<+\infty . \\
\left|\breve{l}_{\mu}(r, R)-l_{\mu}(r, R)\right|=O(1)
\end{array}\right.
$$

Moreover, for all fixed numbers $a \in(0,1], b \in[1,+\infty)$ we have

$$
\left\{\begin{array}{l}
\left|l_{\mu}^{\mathrm{rh}}(r, R)-l_{\mu}^{\mathrm{rh}}(a r, b R)\right|=O(1) \\
\left|l_{\mu}^{\mathrm{h}}(r, R)-l_{\mu}^{\mathrm{lh}}(a r, b R)\right|=O(1) \\
\left|l_{\mu}(r, R)-l_{\mu}(a r, b R)\right|=O(1)
\end{array} \quad \text { for all } \quad r_{0} \leqslant r<R<+\infty .\right.
$$

Proof. Relations (3.14) follow (3.12) since for all $r_{0}>0$ and measures $\mu$ of a finite upper density the relations hold

$$
\left|\frac{\mu\left(R ; \cos ^{ \pm}\right)}{R}-\frac{\mu\left(r ; \cos ^{ \pm}\right)}{r}\right| \leqslant \frac{|\mu|^{\mathrm{rad}}(R)}{R}+\frac{|\mu|^{\mathrm{rad}}(r)}{r}=O(1) \quad \text { as } \quad r_{0} \leqslant r<R<+\infty .
$$

Relations (3.15) are implied by (3.14) and the inequality

$$
\left|\breve{l}_{\mu}^{\mathrm{rh}}(a r, b R)-\breve{l}_{\mu}^{\mathrm{rh}}(r, R)\right| \leqslant \sup _{a r<t \leqslant r} \frac{|\mu|^{\mathrm{rad}}(t)}{t} \ln \frac{1}{a}+\sup _{R<t \leqslant b R} \frac{|\mu|^{\mathrm{rad}}(t)}{t} \ln b,
$$

where in the left hand side one can replace $\breve{l}^{\text {rh }}$ by $\breve{l}^{\mathrm{h}}$.

Remark 3. According Proposition 3, relations (3.14) and Remark 1, various logarithmic measures and submeasures of intervals (1.7 ), (1.7l), $1.7 \mathrm{~m})$ in Definition 2 beginning with $l$ can be replaced by corresponding them in view of (3.14) functions of intervals (3.13r), (3.13 . (3.13m beginning with $\breve{l}$ in conclusion (1.10) of Theorem 1; we employ the same terminology for them. In what follows, we identify these two equivalent up to an additive constant logarithmic measures and submeasures of the intervals for the measures of an upper finite density and we shall denote them as in Definition 1.7 with no accent ${ }^{\sim}$ over $l$.

Definition 4 ([18], developing of [3, Def. 3.4,3.5]). Let $0<r_{0} \in \mathbb{R}^{+}$, $l$ be a function of intervals $(r, R] \subset r_{0}+\mathbb{R}^{+}$with values in $\mathbb{R}_{ \pm \infty}, l(r, R):=l((r, R])$. For $l$ we define four logarithmic block-densities:

$$
\begin{aligned}
& \ln -\overline{\operatorname{dens}}(l):=\limsup _{a \rightarrow+\infty} \frac{1}{\ln a} \limsup _{r \rightarrow+\infty} l(r, a r) ; \\
& \ln \underline{\operatorname{dens}}(l):=\liminf _{a \rightarrow+\infty} \frac{1}{\ln a} \limsup _{r \rightarrow+\infty} l(r, a r) \text {; }
\end{aligned}
$$

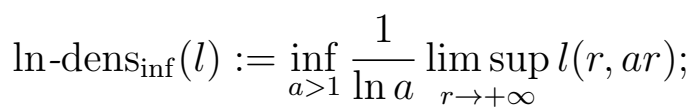

$$
\begin{aligned}
& \ln -\operatorname{dens}_{\mathrm{b}}(l):=\inf \left\{b \in \mathbb{R}^{+}: \sup _{r_{0} \leqslant r<R<+\infty}\left(l(r, R)-b \ln \frac{R}{r}\right)<+\infty\right\} .
\end{aligned}
$$

A function of intervals $l \geqslant 0$ is called logarithmic submeasure of intervals in the vicinity of $+\infty$ if for some number $r_{0}>0$ two conditions hold:

[11] $\sup _{r \geqslant r_{0}} l(r, 2 r)<+\infty$ (logarithmic growth);

[12] $l\left(r_{1}, r_{3}\right) \leqslant l\left(r_{1}, r_{2}\right)+l\left(r_{2}, r_{3}\right)$ for all $r_{0} \leqslant r_{1}<r_{2}<r_{3}<+\infty$ (subadditivity).

If in the first inequality in [2] for some $r_{0}>0$ the sign $\leqslant$ can be replaced by $=$ for all $r_{0} \leqslant r_{1}<r_{2}<r_{3}<+\infty$ (additivity), then the function of intervals $l$ is called logarithmic measure of intervals in the vicinity of $+\infty$. 
Definition 4 implies easily the following statements.

Proposition 4. If $c \in \mathbb{R}^{+}$, and $l_{1}, l_{2}$ are logarithmic submeasures of intervals, then $c l_{1}$, $l_{1}+l_{2}$ and $\max \left\{l_{1}, l_{2}\right\}$ are logarithmic submeasures of intervals.

Proposition 5. For the functions $q_{0}$ in (1.8p) under the condition

$$
\limsup _{|y| \rightarrow+\infty} \frac{q_{0}(y)}{|y|}<+\infty
$$

and for the functions $q$ in (1.8 $\mathrm{q}$ ) and for $q_{E}$ in $(1.9 \mathrm{E})$, the integrals $J_{\mathbb{R}}\left(r, R ; q_{0}\right), J_{\mathbb{R}}(r, R ; q)$, $J_{\mathbb{R}}\left(r, R ; q_{E}\right)$ are logarithmic measures of intervals $(r, R] \subset \mathbb{R}^{+}$. If $\mu \in$ Meas $^{+}$is a measure of a finite upper density, then $l_{\mu}^{\mathrm{rh}}$ and $l_{\mu}^{\mathrm{lh}}$ from (1.7) and (1.7), and also $\breve{l}_{\mu}^{\mathrm{rh}}$ and $\breve{l}_{\mu}^{\mathrm{hh}}$ from (3.13F) and (3.13) are logarithmic measures of intervals, and $l_{\mu}$ from $1.7 \mathrm{~m}$ and $\breve{l}_{\mu}$ from $3.13 \mathrm{~m}$ are logarithmic submeasures of intervals.

Proposition 6 ([18, Thm. 1]). For a logarithmic submeasure of intervals $l \geqslant 0$ all four logarithmic block-densities in (3.16) are finite and coincide, and the upper limit limsup in 3.16 and the lower limit $\liminf _{a \rightarrow+\infty}$ in 3.16 can be replaced by the usual limit $\lim _{a \rightarrow+\infty}$. In what follows for the logarithmic submeasure of intervals $l \geqslant 0$ all four logarithmic block-densities in (3.16) are universally denoted by $\ln -\operatorname{dens}(l)$.

In terms of logarithmic block-density ln-dens the following statement holds.

Theorem 2. Assume that conditions (1.8) and (1.9) of Theorem 1 and condition (3.17) hold. Moreover, let for the function $Q(y):=q(y)+q(-y)$ from (2.16) or from (3.1), one of the conditions (i) or (ii) with property (3.2) in Proposition 1 is satisfied and also

$$
\ln -\operatorname{dens}\left(J_{\mathbb{R}}\left(\cdot, \cdot, q_{0}+q+q_{E}\right)\right)=0 .
$$

Then

$$
\ln -\operatorname{dens}\left(l_{\nu}\right) \leqslant \min \left\{\ln -\operatorname{dens}\left(l_{\mu}^{\mathrm{rh}}\right), \ln -\operatorname{dens}\left(l_{\mu}^{\mathrm{lh}}\right)\right\} \leqslant \ln -\operatorname{dens}\left(l_{\mu}\right) .
$$

Proof. Under additional conditions (ii) or (ii) with property (3.2) in Proposition 1, conclusion 1.10 of Theorem 1 becomes conclusion (3.4) of Proposition 1, which, in particular, for each $a>1$ can be written as

$$
l_{\nu}(r, a r) \leqslant \min \left\{l_{\mu}^{\mathrm{rh}}(r, a r), l_{\mu}^{\mathrm{lh}}(r, a r)\right\}+C J_{\mathbb{R}}\left(r, a r ; q_{0}+q_{E}+q\right)+C \quad \text { for all } r \geqslant r_{0} .
$$

Then, passing to limit as $r$ tends to $+\infty$, we obtain

$$
\begin{aligned}
\limsup _{r \rightarrow+\infty} l_{\nu}(r, a r) \leqslant & \min \left\{\limsup _{r \rightarrow+\infty} l_{\mu}^{\mathrm{rh}}(r, a r), \limsup _{r \rightarrow+\infty} l_{\mu}^{\mathrm{lh}}(r, a r)\right\} \\
& +C \limsup _{r \rightarrow+\infty} C J_{\mathbb{R}}\left(r, a r ; q_{0}+q_{E}+q\right)+C,
\end{aligned}
$$

Dividing both sides of the latter inequality by $\ln a$ and passing to the limit as $a$ tends to $+\infty$, in notations of Definition (3.16- we obtain the following chain of inequalities and identities:

$$
\begin{gathered}
\ln -\overline{\operatorname{dens}}\left(l_{\nu}\right) \leqslant \min \left\{\ln -\overline{\operatorname{dens}}\left(l_{\mu}^{\mathrm{rh}}\right), \ln -\overline{\operatorname{dens}}\left(l_{\mu}^{\mathrm{hh}}\right)\right\}+C \ln -\overline{\operatorname{dens}} J_{\mathbb{R}}\left(\cdot, \cdot ; q_{0}+q_{E}+q\right) \\
\stackrel{3.18}{=} \min \left\{\ln -\overline{\operatorname{dens}}\left(l_{\mu}^{\mathrm{rh}}\right), \ln -\overline{\operatorname{dens}}\left(l_{\mu}^{\mathrm{lh}}\right)\right\} \stackrel{1.7 \mathrm{~h}}{\leqslant} \ln -\overline{\operatorname{dens}}\left(l_{\mu}\right) .
\end{gathered}
$$

By assumption 6 on coinciding all four logarithmic block-densities in Definition (3.16), this gives exactly 3.19 .

For zero entire function of exponential type 0, by the definition, the set of its zeroes is Zero $_{0}=\mathbb{C}$, and the counting measures reads as $n_{\text {Zero } 0}(S) \stackrel{\sqrt[1.6]{=}}{=}+\infty$ for each $S \subset \mathbb{C}$. In particular, $\ln -\operatorname{dens}\left(l_{\text {Zero }}\right)=+\infty$. 
We mention the uniqueness theorem for entire functions of exponential type implied by Theorem 1.

Theorem 3. Let $\mathbf{Z}=\left\{\mathbf{z}_{k}\right\}_{k=1,2, \ldots} \subset \mathbb{C}$ be a sequence of complex points of an upper finite density in the sense of 1.12 , that is,

with the logarithmic submeasure of intervals

$$
n_{\mathrm{Z}}^{\mathrm{rad}}(r) \stackrel{1.6}{=} n_{\mathrm{Z}}(D(r))=O(r) \quad \text { as } \quad r \rightarrow+\infty
$$

$$
l_{\mathrm{Z}}(r, R):=\max \left\{\sum_{\substack{r<\left|z_{k}\right| \leqslant R \\ \operatorname{Re} \mathbf{z}_{k}>0}} \operatorname{Re} \frac{1}{\mathrm{z}_{k}}, \sum_{\substack{r<\left|z_{k}\right| \leqslant R \\ \operatorname{Re} z_{k}<0}} \operatorname{Re}\left(-\frac{1}{\mathbf{z}_{k}}\right)\right\} \stackrel{1.7}{=} l_{n_{\mathbf{Z}}}(r, R) .
$$

Let conditions $11.8 \mathrm{M}),(1.8 \mathrm{p}),(1.8 \mathrm{q}),(1.9 \mathrm{E}),(3.17)$ be satisfied and an entire function of exponential type $f$ vanishes on $\mathrm{Z}$ in the sense that $n_{\text {Zero }_{f}} \geqslant n_{\mathrm{Z}}$ and for each $y \in \mathbb{R}^{+} \backslash E$, the inequality holds:

$$
\ln |f(i y) f(-i y)| \leqslant \mathrm{C}_{M}(i y, q(y))+\mathrm{C}_{M}(-i y, q(-y))+q_{0}(y)+q_{0}(-y) .
$$

If (3.18) holds and at that $\ln$-dens $\left(l_{\mathrm{Z}}\right)>\ln$-dens $\left(J_{i \mathbb{R}}(\cdot, \cdot ; M)\right)$, then $f=0$.

Proof. Assume that $f \neq 0$ and let $u:=\ln |f|$. Then $(1.8 \mathrm{u})$ holds with $\nu:=n_{\mathrm{Z}}$. It follows from (3.20), Proposition 1 with $a>1$ and (3.4) that

$$
l_{Z}(r, a r)=l_{n_{\mathrm{Z}}}(r, a r) \leqslant J_{i \mathbb{R}}(r, a r ; M)+C J_{\mathbb{R}}\left(r, a r ; q_{0}+q_{E}+q\right)+C \quad \text { as } \quad r \geqslant r_{0} .
$$

Passing to the limit as $r$ tends to $+\infty$, dividing then by $\ln a$ and passing to the limit as $a$ tends to $+\infty$, by Proposition 5, Definition (3.16) and (3.18) we obtain

$$
\ln \text {-dens }\left(l_{\mathrm{Z}}\right)=\ln \overline{-\operatorname{dens}}\left(l_{\mathrm{Z}}\right) \leqslant \ln \overline{\operatorname{dens}}\left(J_{i \mathbb{R}}(\cdot, \cdot ; M)\right)=\ln \text {-dens }\left(J_{i \mathbb{R}}(\cdot, \cdot ; M)\right) .
$$

By Proposition 6 this contradicts the condition $\ln$-dens $\left(l_{\mathrm{Z}}\right)>\ln$-dens $\left(J_{i \mathbb{R}}(\cdot, \cdot ; M)\right)$.

\section{BIBLIOGRAPHY}

1. W.K. Hayman, P.B. Kennedy. Subharmonic functions. Academic Press, London (1976).

2. Th. Ransford. Potential Theory in the Complex Plane, Cambridge University Press, Cambridge (1995).

3. P. Malliavin, L.A. Rubel. On small entire functions of exponential type with given zeros // Bull. Soc. Math. France. 89:2, 175-201 (1961).

4. L.A. Rubel (with J.E. Colliander). Entire and meromorphic functions. Springer, New York (1996).

5. I.F. Krasichkov-Ternovskii. Invariant subspaces of analytic functions. II. Spectral synthesis of convex domains // Matem. Sborn. 88(130):1(5), 3-30, (1972). [Math. USSR-Sb. 17:1, 1-29 (1972).]

6. B.N. Khabibullin. Smallness of the growth on the imaginary axis of entire functions of exponential type with given zeros // Matem. Zamet. 43:5, 644-650 (1988). [Math. Notes. 43:5, 372-375 (1988).]

7. B.N. Khabibullin. On the growth of entire functions of exponential type near a straight line // Matem. Zamet. 70:4, 621-635 (2001). [Math. Notes. 70:4, 560-573 (2001)].

8. B.N. Khabibullin. On the growth of exponential-type entire-functions along the imaginary axis // Dokl. AN SSSR. 302:2, 270-273 (1988). (in Russian).

9. B.N. Khabibullin. On the growth of entire functions of exponential type along the imaginary axis // Matem. Sbornik. 180:5, 706-719 (1989). [Math. USSR-Sbornik 67:1, 149-163 (1990).]

10. B.N. Khabibullin. On the growth of the entire functions of exponential type with given zeros along a line // Anal. Math. 17:3, 239-256 (1991). (in Russian).

11. B.N. Khabibullin. Distribution of zeroes of entire functions and balayage // Habil. Thesis. Ufa (1992). 
12. B.N. Khabibullin. Completeness of exponential systems and uniqueness sets. 4th ed. Bashkir State University, Ufa (2012). (in Russian).

13. B.N. Khabibullin, A.V. Shmeleva. Balayage of measures and subharmonic functions to a system of rays. I: The classical case // Alg. Anal. 31:1, 156-210 (2019). [St. Petersbg. Math. J. 31:1, 117-156 (2020).]

14. N.S. Landkof. Foundations of modern potential theory. Nauka, Moscow (1966). [Springer, Berlin (1972).]

15. V.S. Azarin. Growth theory of subharmonic functions. Birkhäser, Basel (2009).

16. A.F. Grishin, T.I. Malyutina. New formulae for indicators of subharmonic functions // Matem. Fiz. Anal. Geom. 12:1, 5-72 (2005). (in Russian).

17. B.N. Khabibullin, A.V. Shmeleva, Abdullina Z.F. Balayage of measures and subharmonic functions to a system of rays. II. Balayage of finite kind and regularity of growth on one ray // Alg. Anal. 32:1, 208-243 (2020). (in Russian).

18. M.R. Karimov, B.N. Khabibullin. Coincidence of some distribution densities of set and completeness of systems of entire functions // in Proc. Int. Conf. "Complex analysis, differential equations and related issues". III, Ufa, 29-34 (2000). (in Russian).

Anna Evgenievna Salimova,

Bashkir State University,

Zaki Validi str. 32,

450076, Ufa, Russia

E-mail: anegorova94@bk.ru

Bulat Nurmievich Khabibullin,

Bashkir State University,

Zaki Validi str. 32,

450076, Ufa, Russia

E-mail: khabib-bulat@mail.ru 